\title{
Chronochemistry in neurodegeneration
}

\section{Annalisa Pastore* and Salvatore Adinolfi}

Department of Clinical Neuroscience, Institute of Psychiatry, King's College London, London, UK

\author{
Edited by: \\ James Olcese, Florida State \\ University College of Medicine, USA

\section{Reviewed by:} \\ Erik Maronde, University of Frankfurt, \\ Germany \\ Baojin Ding, University of \\ Massachusetts Medical School, USA \\ *Correspondence: \\ Annalisa Pastore, Department of \\ Clinical Neuroscience, Institute of \\ Psychiatry, King's College London, \\ De'Crespigny Park, London SE5 8AF, \\ UK \\ e-mail:apastor@nimr.mrc.ac.uk
}

The problem of distinguishing causes from effects is not a trivial one, as illustrated by the science fiction writer Isaac Asimov in a novel dedicated to an imaginary compound with surprising "chronochemistry" properties. The problem is particularly important when trying to establish the etiology of diseases. Here, we discuss how the problem reflects on our understanding of disease using two specific examples: Alzheimer's disease (AD) and Friedreich's ataxia (FRDA). We show how the fibrillar aggregates observed in AD were first denied any interest, then to assume a central focus, and to finally recess to be considered the dead-end point of the aggregation pathway. This current view is that the soluble aggregates formed along the aggregation pathway rather than the mature amyliod fiber are the causes of disease, Similarly, we illustrate how the identification of causes and and effects have been important in the study of FRDA. This disease has alternatively been considered as the consequence of oxidative stress, iron precipitation or reduction of ironsulfur cluster protein context. We illustrate how new tools have recently been established which allow us to follow the development of the disease. We hope that this review may inspire similar studies in other scientific disciplines.

Keywords: Alzheimer's disease, determinism, disease development, Friedreich's ataxia

\section{INTRODUCTION}

\section{THE IMPACT OF DETERMINISM IN OUR LIVES}

By philosophical choice or from our simple and most direct perception of reality, we are accustomed to consider the world as deterministic, i.e., as being ruled and regulated by the principle of causality. This principle states that each action or event is the consequence of a previous action or event. We cannot have the effect before the cause. We have plenty of everyday examples: being hit by a car while crossing a road implies that a car was driving along the road and run over a person who was crossing the road. Surely we could not have a car accident before the car's arrival. Likewise, if a cake comes out from the oven this necessarily means that someone must have baked it and could not be the other way around.

Occasionally, some dreamers have considered the possibility that determinism could be violated and explored how this possibility could reflect on our life. A paradigmatic example is that of the scientist and science fiction writer Isaac Asimov, who wrote a spoof of the deterministic concept in a novel dedicated to thiotimoline (Asimov, 1948). This is supposed to be a wonder substance derived from the bark of the (fictitious) shrub Rosacea karlsbadensis rufo. Thiotimoline has the property of solubilising exactly $1.2 \mathrm{~s}$ (not a second before or after) before the researcher adds water to the compound, thus challenging the principle of cause and effect as in this case the effect would precede the cause... The author's explanation for such extraordinary behavior is chemically "rigorous" although highly fictional: in the thiotimoline molecule, there is at least one carbon atom that has two of the four chemical bonds in the normal space and time dimensions; one of the other two bonds projects into the future and the other into the past, thus bridging time. As imagined by Asimov, if true, the consequences would be unimaginable: the substance could be used to create fantastic mechanisms that could be exploited in "chronochemistry," a discipline of Asimov's invention which only Russian scientists excel in while the Americans, more suspicious, are unfortunately left behind...

Letting aside this and similar fictional possibilities, the concepts of determinism and causality remain simple and undeniable certainties that rule our everyday lives.

Where things go fuzzier is when we do not know the events that well. Not that we would doubt their deterministic nature but there might be cases in which it is difficult to distinguish between cause and effect. The concept acquires a dramatic importance when referred to medicine, a field where the problem of identifying the correct causality is directly related to diagnostics. Failure to identify the cause, or confusion between cause and effect, can direct the cure along false paths making it ineffective, if not dangerous. A heavy headache and stomach sickness could well be explained by assuming that having developing a headache we started also having an upset stomach. But it is equally possible that because of feeling sick we developed headache or even that the two events are uncorrelated and that the two pathological conditions came out at the same time, perhaps as the effect of a third event such as a viral attack or any other direct or indirect cause.

There are in principle three criteria that need to be met before we can say that there is a causal relationship between two events. First, we must be able to show that there is a temporal sequence of events and that the cause happened before the effect. Second, there should be co-variations of the cause and the effect: if one parameter influencing one event was increased, the other event should be affected in a simple binary relationship. Finally, it should be possible to rule out plausible alternative explanations that could explain the co-detection of the two events. Most often, establishing 
these three criteria is far from being easy, especially when we wish to apply them to biology or medicine.

In this review, we discuss the difficulties of identifying cause and effects in specific biological events that are directly related to neurodegeneration and the problem of identifying disease etiology. We show how the problem can be clarified by the possibility of identifying a clear "time zero" (i.e., the time point when the chain of events causing the disease starts) and by checking how the symptoms accumulate from this point. We shall consider two paradigmatic examples: the case of the amyloid-related Alzheimer's disease (AD) and the example of Friedreich's ataxia (FRDA), two diseases studied widely in the last few years. We shall also discuss the implications that these specific examples have for our understanding of the biological phenomena causing disease.

\section{THE PROBLEM OF ESTABLISHING CAUSES AND EFFECTS IN DISEASE ETIOLOGY}

Alzheimer's disease is an aging-related disease of increasing impact for the world population which involves impairment of memory, reasoning, abstraction, and language (Christensen et al., 2009). The cytological and biochemical complexity of this disease has made it difficult to find an easy agreement on the temporal sequence of events that lead to $\mathrm{AD}$ and, as a consequence, on the steps most amenable to drug treatment. AD was first diagnosed and studied by the Bavarian psychiatrist Alois Alzheimer, who identified the presence of unusual formations in the post-mortem brain of a 51 year old patient (Alzheimer, 1907). These lesions are known as amyloid plaques as amyloid plaques and neurofibrillar tangles according to their morphology and location (Selkoe, 2011; Serrano-Pozo et al., 2011). Only much later, it became clear that the lesions are proteinaceous formations that mainly contain, respectively, $A \beta$ peptides that are degradation products of the "amyloid precursor protein" (or APP) (Kang et al., 1987) and the microtubule-associated protein (MAP) tau protein (Brion et al., 1985; Masters and Selkoe, 2012).

We now know that these two molecules are intrinsically unstructured polypeptides that are highly hydrophobic and have a high tendency to misfold and form fibrillar aggregates termed amyloids from their tintorial properties. It is accepted that amyloid fibers adopt a $\beta$-rich structure of the same type as that observed in silk (Marshall and Serpell, 2009). The conversion of $\alpha$-helix or random coil conformations within normally soluble proteins into $\beta$-sheet rich assemblies is a common theme recurrent in several neurodegenerative diseases and has been shown to be a universal solution of the energy landscape common to most if not all proteins (Eichner and Radford, 2011; Ma and Nussinov, 2012).

Interestingly, the presence of plaques and tangles was interpreted differently through time. When Glenner and Wong (1984) first identified $\mathrm{A} \beta$ from post-mortem meninges of $\mathrm{AD}$ patients, the authors suggested that these formations could "provide a diagnostic test for AD and a means to understand its pathogenesis." Other scientists argued instead that the plaques and tangles might "only" be end-stage lesions and that would provide little useful information about etiology and early pathogenesis. Nevertheless, plaques and tangles have been used as diagnostic tools for several decades. As a consequence many people adopted what is known as the "amyloid hypothesis," which assumes that extracellular deposits of
$\mathrm{A} \beta$ peptides are the fundamental cause of $\mathrm{AD}$ (Hardy and Allsop, 1991; Mudher and Lovestone, 2002; Luheshi et al., 2008). Inspired by these studies, amyloid fibers have been thoroughly studied and large attention has been paid to them not only in the AD field but in several other, otherwise uncorrelated diseases also associated to protein aggregates (Luheshi et al., 2008).

Association does not however, necessarily imply a causal relationship even though this inference is rather tempting. More recently, it has become progressively clear that $\mathrm{AD}$ could not after all be caused by the final mature amyloid fibers but by other species which form along the aggregation pathway. It has been suggested that the formation of amyloid fibers could even be a defense mechanism of the cell to protect itself from other more toxic species which have recently been identified in the so called oligomers (Hardy and Selkoe, 2002). These are assembly species formed along the aggregation pathway but well before the mature fibers are formed. The importance of the oligomers rather than mature fibers is supported by several lines of evidence.

Some patients develop severe symptoms well before plaques are formed or at least are detectable. Conversely, plaques can be found in the cortex of aged subjects who are at least apparently healthy, although it is possible that they might have not been tested for subtle cognitive dysfunctions. Oligomers, that is all forms that remain soluble after high-speed ultracentrifugation of brain extracts and not of fibrillar forms, are detectable in buffer-soluble extracts of post-mortem AD patients' cortex (Shankar et al., 2008). These species are cytotoxic as shown for the first time by Lambert et al. (1998). Later on, injection of oligomeric (dimers) species into the cerebral ventricles of healthy adult rats confirmed that they transiently impair the memory of a learned behavior (Shankar et al., 2008). A $\beta$ oligomers have been shown to cause neuronal death in culture and to block long-term synaptic depression (Lambert et al., 1998; Stine et al., 2003; Wang et al., 2004). There is a statistical correlation between cortical levels of soluble $A \beta$ and the extent of synaptic loss and severity of cognitive symptoms (Kuo et al., 1996; Lue et al., 1999; McLean et al., 1999; Wang et al., 1999). Oligomers of the size of $A \beta$ dimers but not monomeric species from the same brains were shown to facilitate long-term synaptic depression in the hippocampus and to decrease dendritic spine density (Shankar et al., 2008; Li et al., 2009b).

Taken together, these findings support the hypothesis that small, soluble oligomers of human $A \beta$ peptides are sufficient to induce several features of the $\mathrm{AD}$ phenotype, including synaptic loss, tau hyper-phosphorylation, neurofibrillary degeneration, and memory impairment, even in the absence of amyloid plaques. When we determine the presence of plaques, in fact, most of the damages have already taken place. Thus the fibrillar aggregates could be the ultimate effect, the escape and not the cause of $\mathrm{AD}$ (Figure 1).

The lesson we may learn from this story could be a hard one. It could be argued that confusion between cause and effect has been negative and has distracted researchers from the "real thing," encouraging them to follow the wrong pathway. At least 10 years have been dedicated to a thorough study of the morphological, mechanical and structural properties of amyloids. However, we can convincingly argue back that it has not been a waste of time in the broader perspective of our fundamental knowledge 


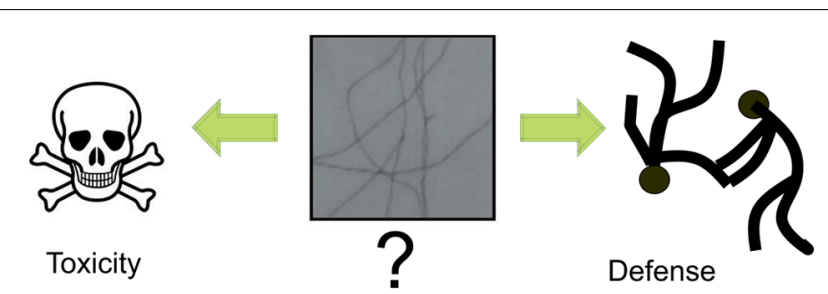

FIGURE 1 | Debated role of fibrillar amyloids. A recurrent question which seems to become clear only recently is whether the fibrillar amyloid species observed in patients' brains are the toxic species or rather a defense mechanism of the cell. The current view is that the toxic species are the soluble aggregates formed through the aggregation process. As such, the mature fibrillar fibres are the final point of this process.

of reality. It is now clear that amyloids are not "just" a rare or totally unwanted structure. Amyloid peptides are routinely found in hormones (Maji et al., 2009) and are produced by E. coli and other gram-negative enteric bacteria (Wang et al., 2008) and by the gram-positive bacterium S. coelicolor (Capstick et al., 2011). While some of the interest in amyloid fibers could be considered ineffective and dangerously delaying an anti-Alzheimer treatment, our "detour" into the amyloid world will certainly prove highly beneficial for many other areas of Biology in the close future.

\section{THE IMPORTANCE OF DETECTING THE VERY EARLY EVENTS OF A DISEASE}

Let's now consider the example of FRDA. This is a neurodegenerative disease caused by an abnormal expansion of a non-coding GAA triplet repeat in the first intron of the FRDA gene (Pastore and Puccio, 2013). This event causes lower expression of the FRDA gene product, the protein frataxin, through heterochromatization of the locus. The age of disease onset correlates inversely with the number of repeats. FRDA provides a unique example (of still ongoing research) in which causes and effects can easily be mixed up until the "right" experiments are designed to establish a temporal relationship between observations.

A large body of work has been produced since the discovery of the gene responsible for this ataxia (Campuzano et al., 1996). We now know that frataxin is a nuclearly encoded protein, produced in the cytoplasm and then imported into and matured in the mitochondrion where its primary function resides. To reach this conclusion it was noticed early on that only eukaryotic frataxins contained an $\mathrm{N}$-terminal extension that could represent an import signal (Gibson et al., 1996). It was also noticed that the distribution of the frataxin gene in different organisms is fully consistent with the fusion event that leads to the formation of mitochondria. The hypothesis was soon after independently proven experimentally (Campuzano et al., 1997).

But what is the role of frataxin in mitochondria? One of the early observations was that of iron deposits in heart tissues of FRDA patients (Sanchez-Casis et al., 1977). This was confirmed by a deletion mutant in yeast $(\triangle \mathrm{YFH} 1)$ that showed a ten-fold increase in the iron concentration in mitochondria and an increase of hypersensitivity to oxidative stress (Babcock et al., 1997). A more complete investigation of the molecular defects present in patients carried out by Rustin and his group identified a deficient activity of iron-sulfur (FeS) cluster-containing subunits of mitochondrial respiratory complexes I, II, III and aconitase in endomyocardial biopsies (Roting et al., 1997). The authors made an attempt to create a temporal connection between the observed strong and yet very different phenotypes. They concluded that iron overload is the "causa prima" that generates oxidative stress through iron-catalyzed Fenton chemistry. This would in turn give rise to oxygen free radicals and iron precipitates. The latter are well known to target efficiently FeS proteins causing them to quickly lose their activities as observed in patients and yeast-null mutants generating the pathology. The authors did not, however, provide any indication of where iron could come to the equation which left the explanation somewhat unsatisfactory.

To address the question in a different way, several laboratories developed animal and cellular models that could allow the study of the multiple effects generated by the reduced production of frataxin as observed in disease. These studies rely on the high conservation of the frataxin gene along evolution, which makes possible to model the effects of frataxin deletion in different systems and compare the effects to those observed in patients' cells. A homozygous mouse model in which the frataxin gene was drastically switched off was the first attempt to understand the primary events caused by the absence of the protein and the cellular phenotype of FRDA (Cossée et al., 2000). This homozygous deletion caused embryonic lethality a few days after implantation, indicating that frataxin is an essential protein in mammals. Surprisingly, though, no iron overload or iron precipitates were detected in degenerating embryos, in open contrast with the hypothesis that iron precipitates are the primary cause of the pathology, thus suggesting that they could be instead a side-effect. These findings were explained assuming that yeast frataxin could have a ferritin-like function as an iron storage chaperone within the mitochondria (Isaya et al., 2000). Cell death in mouse embryos would be the consequence of the lack of a scavenger rather than the result of iron precipitation, reinforcing the idea that iron deposits are a secondary event.

It is important however, to consider that the discrepancy between the data in yeast, in patients and in transgenic mice could be more simply explained by the unfair comparison of conditions detected at very different time points of the disease course. Iron precipitation is the most striking phenomenon observed in patients' tissues, where the disease is usually present for years, and in yeast-null mutants grown for many generations before been used in experiments. In embryos instead, iron accumulation could hardly have time to build up, thus giving a better description of what the cause and the effects may be. Embryos could in facts be an ideal system to capture the early events close to what we shall call "time zero," that is the disease onset.

To better understand the time relationship between events, different laboratories developed conditional knock-out mice for the frataxin gene (Puccio et al., 2001; Martelli et al., 2012) or yeast models in which the gene was put under a galactose-regulated promoter in yeast (Muhlenkhoff et al., 2002). Both systems would allow switching off the gene at a given time point so that this could be taken as "time zero" from which the effects could be followed with controlled molecular and functional assays. To obtain the 
same effect in Drosophila, an RNAi-mediated-suppression strategy was used (Anderson et al., 2005; Llorens et al., 2007). These models all revealed that frataxin deficiency primarily leads to strong defects in FeS cluster containing proteins and FeS cluster maturation followed by accumulation of iron deposits. In yeast, a defect in respiratory efficiency was also detected (Muhlenkhoff et al., 2002), following the disruption of FeS cluster maturation. In mouse, the FeS cluster deficit is observed before the first evidence of a cardiac dysfunction (Martelli et al., 2012). Interestingly, no evidence of oxidative damage was observed in this and in a Drosophila model (Anderson et al., 2005; Llorens et al., 2007). This observation led to the still open question of whether formation of oxygen free radicals (ROS) is at all essential to generate the cellular phenotype associated with FRDA.

The experiments described above were excellent attempts to generate animal models in which frataxin production could be controlled and lowered "to induce the disease," starting the observation of the molecular and cellular phenotype at a well-defined time. However, although conceptually correct, such studies were often carried out without any attempt to investigate the actual time course of events: the phenotype was tested only at few time points which were often spaced different weeks apart. Unfortunately, as in $\mathrm{AD}$, when the phenotype becomes observable most of the events that lead to it have already taken place.

A more direct approach became possible when the proteins associated with the FeS cluster biogenesis machine were purified, both in eukaryotes and prokaryotes (Adinolfi et al., 2009; Prischi et al., 2010). This allowed the possibility of reproducing the FeS cluster biogenesis machine in vitro and of studying the effect of frataxin on the formation of de novo synthesized FeS clusters without the complexity of the in vivo/in cell environments. What makes these experiments particularly attractive is also that the time course of the events can be followed directly and in a continuous way. Data are now available for the bacterial (Adinolfi et al., 2009; Prischi et al., 2010), yeast (Li et al., 2009a), and human (Tsai and Barondeau, 2010; Bridwell-Rabb et al., 2012) systems and have provided a great deal of new information. These studies clearly showed that frataxin and its orthologs play a critical role in regulating FeS biogenesis. These findings were rationalized by the report that frataxin and its orthologues bind specifically and stoichiometrically to the complex Nfs1/Isu (or IscS/IscU in bacteria; Tsai and Barondeau, 2010). These proteins are the two central components of the FeS cluster assembly machine, being respectively the desulphurase that provides sulfur for cluster assembly and the scaffold protein on which the cluster forms.

Much as this approach clarified some issues, it could however, be noticed that the in vivo and in vitro experiments so far described have a completely different time frame for the appearance of the effect of frataxin deficiency letting us wonder whether or how these results can really be compared to each other and reconciled.

To fill the gap and be able to observe and quantify the effects of frataxin in a time frame as close as possible to the in vitro studies, Tamarit and co-workers have recently suggested an alternative approach: they developed a yeast model placing frataxin under a controlled promoter (Moreno-Cermeno et al., 2010). The authors used tetO-YFH1 mutants where the addition of doxycycline to the growth medium allows an efficient and specific repression of frataxin expression. This drug is known not to affect other gene expression. The system was used to follow the effects of frataxin deletion in vivo, starting the measurements of different parameters at the time of addition of doxycycline, and following the time evolution using time-points close to the in vitro experiments (Moreno-Cermeno et al., 2010). Tamarit and co-workers' results indicate that the deregulation of iron metabolism is the primary effect of frataxin depletion, followed by iron accumulation (visible after $4 \mathrm{~h}$ ) that occurs at the early stages. They also demonstrated that increased oxidative damage and decreased concentration of FeS cluster proteins such as aconitase, are late defects visible only after $24 \mathrm{~h}$, and might be considered as secondary effects that are a consequence of iron overload. A possible criticism of this study could be that the residual activity of the FeS cluster proteins after frataxin depletion could be explained by a longer half-life of these proteins. This possibility can be ruled out, since reduction of expression of proteins responsible for FeS cluster biogenesis leads to a more than $80 \%$ reduction of aconitase activity (RodriguezManzaneque et al., 2002; Kumanovics et al., 2008). The presence of normal aconitase activity for $24 \mathrm{~h}$ after frataxin depletion cannot thus be attributed to the initial holo-aconitase pool, but indicates that the FeS cluster machine remains active even in the absence of frataxin.

While more work is still needed to clarify several aspects, the approach proposed by Tamarit together with the in vitro studies provide powerful tools to clarify the temporal pipeline of FRDA and hold a tangible promise for understanding the cellular role of frataxin and its connection to disease (Figure 2).

\section{CONCLUSION}

In this review we have discussed the difficulties of understanding disease etiology, a problem that, if anything, is becoming even

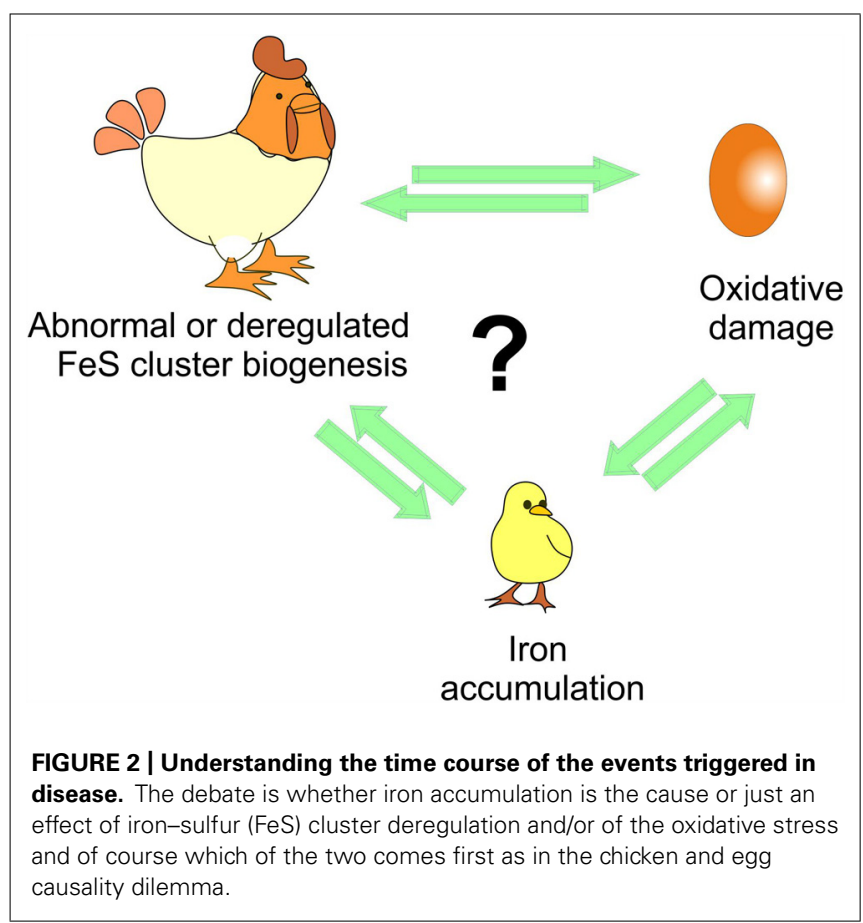


more important in our molecular medicine era. The problem is of particular interest in neuroscience where many diseases are poorly understood and interconnected with several different and apparently uncorrelated symptoms. We discussed two examples in which causes and effects have been confused, AD and the less well known FRDA. The former exemplifies well how the same symptomatology can be considered the aetiological cause or even a defense mechanism which, rather than harming the organism, is activated to prevent further damages. The same concept has been transferred to other neurodegenerative diseases which share with $\mathrm{AD}$ the presence of protein aggregates. FRDA provides a unique example of how experiments can and must be tailored to clarify the time sequence of events. Although still ongoing, the research in this field has made enormous progresses from its early days and holds the promise of unveiling soon the exact mechanism of this still incurable disease.

The take home message that this discussion suggests is that much care needs to be taken before jumping to conclusions and assuming that all factors associated with a disease are its very cause. At the same time, we propose the importance of designing suitable experiments that may clarify the temporal relationship between events as the only way to understand the mechanisms responsible for disease onset.

\section{ACKNOWLEDGMENTS}

We wish to thank Piero Andrea Temussi and Raffaele Pastore for critical reading of the manuscript. This work was supported by the MRC (Grant ref. U117584256) and by the EU EFACTS consortium.

\section{REFERENCES}

Adinolfi, S., Iannuzzi, C., Prischi, F., Pastore, C., Iametti, S., Martin, S., et al. (2009). Bacterial frataxin CyaY is the gatekeeper of iron-sulphur cluster formation catalyzed by IscS. Nat. Struct. Mol. Biol. 4, 390-396. doi: 10.1038/nsmb.1579

Alzheimer, A. (1907). On a peculiar disease of the cerebral cortex. Allgemeine $Z$. Psychiatr. Psychish Gerichtlich Med. 64, 146-148.

Anderson, P. R., Kirby, K., Hiliker, A. J., and Phillips, J. P. (2005). RNAi-mediated suppression of the mitochondrial iron chaperone, frataxin, in Drosophila. Hum. Mol. Genet. 15, 3397-3405. doi: 10.1093/hmg/ddi367

Asimov, I. (1948). "The endochronic properties of resublimated thiotimoline," in Astounding Science Fiction, ed. J. W. Campbell Jr. (New York: Street \& Smith Publ., Inc.), 120-126.

Babcock, M., de Silva, D., Oaks, R., Davis-Kaplan, S., Jiralerspong, S., Montermini, L., etal. (1997). Regulation of mitochondrial iron accumulation by Yfhlp a putative homolog of Frataxin. Science 276, 1709-1712. doi: 10.1126/science.276.5319.1709

Bridwell-Rabb, J., Iannuzzi, C., Pastore, A., and Barondeau, D. (2012). Effector role reversal during evolution: the case of frataxin in Fe-S cluster biosynthesis. Biochemistry 27, 2506-2514. doi: 10.1021/bi201628j

Brion, J. P., Couck, A. M., Passareiro, E., and Flament-Durand, J. (1985). Neurofibrillary tangles of Alzheimer's disease: an immunohistochemical study. J. Submicrosc. Cytol. 17, 89-96.

Campuzano, V., Montermini, L., Lutz, Y., Cova, L., Hindelang, C., Jiralerspong, S., et al. (1997). Frataxin is reduced in Friedreich's ataxia patients and is associated with mitochondrial membrane. Hum. Mol. Genet. 6, 1771-1780. doi: 10.1093/hmg/6.11.1771

Campuzano, V., Montermini, L., Moltò, M. D., Pianese, L., Cossée, M., Cavalcanti, F., et al. (1996). Friedreich's ataxia: autosomal recessive disease caused by an intronic GAA triplet repeat expansion. Science 271, 1423-1427. doi: 10.1126/science.271.5254.1423

Capstick, D. S., Jomaa, A., Hanke, C., Ortega, J., and Elliot, M. A. (2011). Dual amyloid domains promote differential functioning of the chaplin proteins during
Streptomyces aerial morphogenesis. Proc. Natl. Acad. Sci. U.S.A. 108, 9821-9826. doi: 10.1073/pnas.1018715108

Christensen, K., Doblhammer, G., Rau, R., and Vaupel, J. W. (2009). Ageing populations: the challenges ahead. Lancet 374, 1196-1208. doi: 10.1016/S01406736(09)61460-4

Cossée, M., Puccio, H., Gansmuller, A., Koutnikova, H., Dierich, A., LeMeur, M., et al. (2000). Inactivation of the Friedreich ataxia mouse gene leads to early embryonic lethality without iron accumulation. Hum. Mol. Genet. 9, 1219-1226. doi: $10.1093 / \mathrm{hmg} / 9.8 .1219$

Eichner, T., and Radford, S. E. (2011). A diversity of assembly mechanisms of a generic amyloid fold. Mol. Cell 43, 8-18. doi: 10.1016/j.molcel.2011.05.012

Gibson, T. J., Koonin, E. V., Musco, G., Pastore, A., and Bork, P. (1996). Friedreich's ataxin protein: phylogenetic evidence for mitochondrial dysfunction. Trends Neurosci. 19, 465-468. doi: 10.1016/S0166-2236(96)20054-2

Glenner, G. G., and Wong, C. W. (1984). Alzheimer's disease and Down's syndrome: sharing of a unique cerebrovascular amyloid fibril protein. Biochem. Biophys. Res. Commun. 122, 1131-1135. doi: 10.1016/0006-291X(84)91209-9

Hardy, J., and Allsop, D. (1991). Amyloid deposition as the central event in the aetiology of alzheimer's disease. Trends Pharmacol. Sci. 12, 383-388. doi: 10.1016/0165-6147(91)90609-V

Hardy, J., and Selkoe, D. J. (2002). The amyloid hypothesis of Alzheimer's disease: progress and problems on the road to therapeutics. Science 297, 353-356. doi: $10.1126 /$ science. 1072994

Isaya, G., Adamec, J., Rusnak, F., Owen, W. G., Naylor, S., and Benson, L. (2000). Frataxin is an iron storage protein. Am. J. Hum. Genet. 67, 549-562.

Kang, J., Lemaire, H.-G., Unterbeck, A., Salbaum, J. M., Masters, C. L., Grzeschik, K.-M., etal. (1987). The precursor of Alzheimer's disease amyloid A4 protein resembles a cell-surface receptor. Nature 325, 733-736. doi: 10.1038/ $325733 \mathrm{a} 0$

Kumanovics, A., Chen, O. S., Li, L., Bagley, D., Adkins, E. M., Lin, H., et al. (2008). Identification of FRA1 and FRA2 as genes involved in regulating the yeast iron regulon in response to decreased mitochondrial iron-sulphur synthesis. J. Biol. Chem. 283, 10276-10286. doi: 10.1074/jbc.M801160200

Kuo, Y.-M., Emmerling, M. R., Vigo-Pelfrey, C., Kasunic, T. C., Kirkpatrick, J. B., Murdoch, G. H., et al. (1996). Water-soluble A $\beta$ (N-40, N-42) oligomers in normal and Alzheimer disease brains. J. Biol. Chem. 271, 4077-4081. doi: 10.1074/jbc.271.8.4077

Lambert, M. P., Barlow, A. K., Chromy, B. A., Edwards, C., Freed, R., Iosatos, M., et al. (1998). Diffusible, nonfibrillar ligands derived from Abeta1-42 are potent central nervous system neurotoxins. Proc. Natl. Acad. Sci. U.S.A. 95, 6448-6453. doi: 10.1073/pnas.95.11.6448

Li, H., Gakh, O., Smith, D. Y., and Isaya, G. (2009a). Oligomeric yeast frataxin drives assembly of core machinery for mitochondrial iron-sulphur cluster synthesis. J. Biol. Chem. 14, 21971-21980. doi: 10.1074/jbc.M109.011197

Li, S., Hong, S., Shepardson, N. E., Walsh, D. M., Shankar, G. M., and Selkoe, D. (2009b). Soluble oligomers of amyloid $\beta$ protein facilitate hippocampal long-term depression by disrupting neuronal glutamate uptake. Neuron 62, 788-801. doi: 10.1016/j.neuron.2009.05.012

Llorens, J. V., Navarro, J. A., Martinez-Sebastian, M. J., Baylies, M. K., Schneuwly, S., Botella, J. A., et al. (2007). Causative role of oxidative stress in a Drosophila model of Friedreich ataxia. FASEB J. 21, 333-344. doi: 10.1096/fj.05-5709com

Lue, L. F., Kuo, Y. M., Roher, A. E., Brachova, L., Shen, Y., Sue, L., et al. (1999). Soluble amyloid $\beta$ peptide concentration as a predictor of synaptic change in Alzheimer's disease. Am. J. Pathol. 155, 853-862. doi: 10.1016/S0002-9440(10)65184-X

Luheshi, L. M., Crowther, D. C., and Dobson, C. M. (2008). Protein misfolding and disease: from the test tube to the organism. Curr. Opin. Chem. Biol. 12, 25-31. doi: 10.1016/j.cbpa.2008.02.011

Ma, B., and Nussinov, R. (2012). Selective molecular recognition in amyloid growth and transmission and cross-species barriers. J. Mol. Biol. 421, 172-184. doi: 10.1016/j.jmb.2011.11.023

Maji, S. K., Perrin, M. H., Sawaya, M. R., Jessberger, S., Vadodaria, K., Rissman, R. A., et al. (2009). Functional amyloids as natural storage of peptide hormones in pituitary secretory granules. Science 325, 328-332. doi: 10.1126/science.1173155

Marshall, K. E., and Serpell, L. C. (2009). Structural integrity of beta-sheet assembly. Biochem. Soc. Trans. 37, 671-676. doi: 10.1042/BST0370671

Martelli, A., Napierala, M., and Puccio, H. (2012). Understanding the genetic and molecular pathogenesis of Friedreich's ataxia through animal and cellular models. Dis. Model. Mech. 5, 165-176. doi: 10.1242/dmm.008706 
Masters, C. L., and Selkoe, D. J. (2012). Biochemistry of amyloid $\beta$-protein and amyloid deposits in Alzheimer disease. Cold Spring Harb. Perspect. Med. 2:a006262. doi: 10.1101/cshperspect.a006262

McLean, C. A., Cherny, R. A., Fraser, F. W., Fuller, S. J., Smith, M. J., Beyreuther, K., etal. (1999). Soluble pool of $A \beta$ amyloid as a determinant of severity of neurodegeneration in Alzheimer's disease. Ann. Neurol. 46, 860-866. doi: 10.1002/1531-8249(199912)46:6<860::AID-ANA8>3.0 $\mathrm{CO} ; 2-\mathrm{M}$

Moreno-Cermeno, A., Obis, E., Belli, G., Cabiscol, E., Ro, J., and Tamarit, J. (2010). Frataxin depletion in yeast triggers up-regulation of iron transport systems before affecting iron-sulphur enzyme activities. J. Biol. Chem. 285, 41653-41664. doi: 10.1074/jbc.M110.149443

Mudher, A., and Lovestone, S. (2002). Alzheimer's disease-do tauists and baptists finally shake hands? Trends Neurosci. 25, 22-26. doi: 10.1016/S01662236(00)02031-2

Muhlenkhoff, U., Richhardt, N., Ristow, M., Kispal, G., and Lill, R. (2002). The yeast frataxin homolog Yfhlp plays a specific role in the maturation of cellular Fe/S proteins. Hum. Mol. Genet. 11, 2025-2036. doi: 10.1093/hmg/11. 17.2025

Pastore, A., and Puccio, H. (2013). Frataxin: a protein in search for a function. $J$. Neurochem. 126, 43-52. doi: 10.1111/jnc.12220

Prischi, F., Konarev, P. V., Iannuzzi, C., Pastore, C., Adinolfi, S., Martin, S. R., et al. (2010). Structural bases for the interaction of frataxin with the central components of iron-sulphur cluster assembly. Nat. Commun. 1, 95. doi: 10.1038/ncomms 1097

Puccio, H., Simon, D., Cossée, M., Criqui-Filipe, P., Tiziano, F., Melki, J., et al. (2001). Mouse model for Friedreich ataxia exhibit cardiomyopathy, sensory nerve defect and Fe-S enzyme deficiency followed by intramitochondrial iron deposits. Nat Genet. 27, 181-186. doi: 10.1038/84818

Rodriguez-Manzaneque, M. T., Tamarit, J., Belli, G., Ros, J., and Herrero, E. (2002). Grx5 is a mitochondrial glutaredoxin required for the activity of iron/sulphur enzymes. Mol. Biol. Cell 13, 1109-1121. doi: 10.1091/mbc.0110-0517

Roting, A., de Lonlay, P., Chretien, D., Foury, F., Koenig, M., Sidi, D., et al. (1997). Aconitase and mitochondrial iron-sulphur protein deficiency in Friedreich ataxia. Nat. Genet. 17, 215-217. doi: 10.1038/ng1097-215

Sanchez-Casis, G., Cote, M., and Barbeau, A. (1977). Pathology of the heart in Friedreich's ataxia: review of the literature and report of one case. Can. J. Neurol. Sci. 3, 349-354.

Selkoe, J. (2011). Alzheimer's disease. Cold Spring Harb. Perspect. Biol. 3:a004457. doi: $10.1101 /$ cshperspect.a004457
Serrano-Pozo, A., Frosch, M. P., Masliah, E., and Hyman, B. T. (2011). Neuropathological alterations in Alzheimer disease. Cold Spring Harb. Perspect. Med. 1:a006189. doi: 10.1101/cshperspect.a006189

Shankar, G. M., Li, S., Mehta, T. H., Garcia-Munoz, A., Shepardson, N. E., Smith, I., et al. (2008). Amyloid- $\beta$ protein dimers isolated directly from Alzheimer's brains impair synaptic plasticity and memory. Nat. Med. 14, 837-842. doi: $10.1038 / \mathrm{nm} 1782$

Stine, W. B. Jr., Dahlgren, K. N., Krafft, G. A., and LaDu, M. J. (2003). In vitro characterization of conditions for amyloid- $\beta$ peptide oligomerization and fibrillogenesis. J. Biol. Chem. 278, 11612-11622. doi: 10.1074/jbc.M210207200

Tsai, C. L., and Barondeau, D. (2010). Human frataxin is an allosteric switch that activates the Fe-S cluster biosynthetic complex. Biochemistry 49, 9132-9139. doi: 10.1021/bi1013062

Wang, J., Dickson, D. W., Trojanowski, J. Q., and Lee, V. M. (1999). The levels of soluble versus insoluble brain A $\beta$ distinguish Alzheimer's disease from normal and pathologic aging. Exp. Neurol. 158, 328-337. doi: 10.1006/exnr.1999.7085

Wang, Q., Walsh, D. M., Rowan, M. J., Selkoe, D. J., and Anwyl, R. (2004). Block of long-term potentiation by naturally secreted and synthetic amyloid b-peptide in hippocampal slices is mediated via activation of the kinases c-Jun N-terminal kinase, cyclin-dependent kinase 5, and p38 mitogenactivated protein kinase as well as metabotropic glutamate receptor type 5. J. Neurosci. 24, 3370-3378. doi: 10.1523/JNEUROSCI.1633-03.2004

Wang, X., Hammer, N. D., and Chapman, M. R. (2008). The molecular basis of functional bacterial amyloid polymerization and nucleation. J. Biol. Chem. 283, 21530-21539. doi: 10.1074/jbc.M800466200

Conflict of Interest Statement: The authors declare that the research was conducted in the absence of any commercial or financial relationships that could be construed as a potential conflict of interest.

Received: 30 December 2013; accepted: 28 February 2014; published online: 31 March 2014.

Citation: Pastore A and Adinolfi S (2014) Chronochemistry in neurodegeneration. Front. Mol. Neurosci. 7:20. doi: 10.3389/fnmol.2014.00020

This article was submitted to the journal Frontiers in Molecular Neuroscience. Copyright (C) 2014 Pastore and Adinolfi. This is an open-access article distributed under the terms of the Creative Commons Attribution License (CC BY). The use, distribution or reproduction in other forums is permitted, provided the original author(s) or licensor are credited and that the original publication in this journal is cited, in accordance with accepted academic practice. No use, distribution or reproduction is permitted which does not comply with these terms. 